$$
2=-551
$$

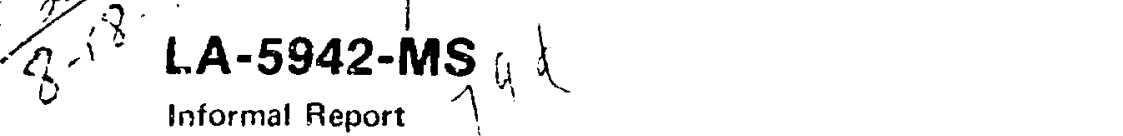

UC. 34 and UC.79d

Reporting Date: April 1975

Issued: June 1975

\title{
Numerical Calculation of Two-Phase Flows
}

by

John R. Trayis

Francis H. Harlow

Anthony A. Amsden 
In the interest of prompt distribution, this report was not edited by the Technical Information staff.

This work supported by the Reactor Safety Research Division. US Energy Research and Development Administration.

Frisled in the United Slate of Americo. Avallable from Notional Technical Informotton Sisvies

US Departmeal of Commerce

5285 Port Royal Rlood

Springthald, VA 22151

Price: Printed Copy 14.00 Mierofich $\$ 2.25$

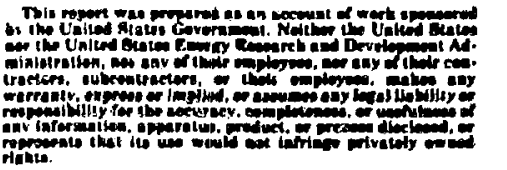

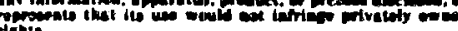




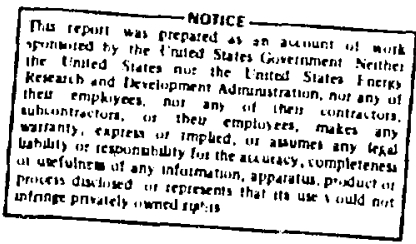

NUMERICAL CALCULATION OF THO-PHASE FLOWS

by

John R. Travis

Francis H. Harlow

Anthony A. Amsden

\section{ABSTRACT}

The theorntical study of time-varying two-phase flow problems in several space dimensions introduces such a complicated set of coupled nonlinear partial differential equations that numerical solution procedures for high-speed computers are required in almost all but the simplest examples. Efficient attainment of realistic solutions for practical problems requires a finite-difference formulation that is simultaneously implicit in the treatment of mass convection, equations of state, and the momentum coupling between phases. We describe such a method, discuss the equations on which it is based, and illustrate its properties by means of examples. In particular, we emphasize the capability for calculating physical instabilities and other time-varying dynamics, at the same time avoiding numerical instability. The computer code is applicable to problems in reactor safety analysis, the dynamics of fluidized dust beds, raindraps or aerosol transport, and a variety of similar circumstances, including the effects of phase transitions and the release of latent heat or chemical energy.

\section{INTROOUCTION}

Some important circumstances of fluid flow involve the interpenetration of several different materials. Examples are the passage of raindrops through air. of bubbles or sediments through water. and of gas through a fluidized dust bed. If the embedded material is a single particle, orop, or bubble. then its dynamics and concurrent reaction back unto the fluid may be amenable to reiatively simple analysis through the use of a drag function for momentun exchange, a phase transition function for mass exchange, and a heat transfer function for energy exchange. If there are numerous separate pieces of the embedded material, then the dynamics must also inciude collective effects, which can complicare the theoretical flow analysis almost to intractability.

The usual procedure for a many-particle analysis is to represent the dynamics of each material by means of field variables. Consider a mixture of air and water in froportions that can vary from small bubbles in a fluid to small droplets in a gas. In each extreme, the disperse material may be closely tied ("frozen in") to the motion of the surrounding contiviuous phase, so that there is no relative motion, or drift, between them. For intermediate proportions, the added complications include the retarded drift of the dispersed phase, the induced currents in the continuous phase, condensation and evaporation from or to the water vapor in the air. the release or absorption of latent heat, and the resultant effects of buoyancy. At the same time, collective effects profoundly modify the dynamics from what would occur if the dispersed bubbles or droplets were independent. Collisions and interacting wakes alter the momentum exchange, change the mean size scale of the dispersed material, and distort the droplets or bubbles to large cepartures from sphericity. The variables necessary to describe 
these interacting processes nust include two different fields of velocity vectors, a void-fraction or porosity function, tho fields of temperature, and two (or possibly one) fields of pressure.

More generally, the effects of a spectrum of size scale for the dispersed phase leads to the requirement for a continuum of rield velocities, or alternatively for a distribution function like the Maxwell-Boltzmann distribution for molecular velocities in a gas. It is easy to see that the compleyities for the analysis of such a system can grow almost without bound, especially if the dispersed phase consists of a variety of different miterials with the potentiality for chemical or nuclear reactions occurring during the dynamics. It is therefore easily realized that rumerical solutions with high-speed computer offer the only opportunity for realistic thecretical analysis of any but the most simple muttiphase flow problems.

The purpose of this report is to describe a recently developed numerical solution procedure called the Implicit, Multi-Field (IMF) technique,' which is applicable to the study of time-varying (initialvalue) problens in several space dimensions, in which the continuous phas: is a liquid or gas flowing at dny Mach number from zero (the incompressibie limit) to well above unity (the supersonic limit), and the dispersed ohase is formed of microscopically incunpressible particles or aroplets all with the same size scale. Because this details of the nimerical echnique and associatad compucer code have been discussec elsownere." this report concenerates on a discussion of the fundarental equations, and un the interpretation and accuracy of the numerical solutions.

in carticular. we show an alternative derivaticn of the momentum equations, which avoids some tifficulties encountered by previous authors in formuldting the pressure-gradient terms. He also discuss the 5tability of the equitions from botl, the continuum and numerical (finise-difference) points of view. In this regard, three aspects of the mattar are of importince:

1. The high-frequency instabilities of the differential equations, which make the formulation "illposed," and cannot in any case, be resolyed by the finite-ditference equations,
2. The luw-trequency instabilities of the ditferential equations, which regresent important phys, ical processes in the time-varying dynamics, and

3. Instabilities that might be introduced by the numerical solution procedure, whose avoidance by implicitness of the formulation or the introduction of dissipation inust not be accomplished at the expense of damping the dymanics of interest.

These topics are discussed from an analy ${ }^{*} i$ i. viewpoint, and illustrated by medns of results from some computer calculations.

\section{STAIISTICAL DFRIVATICN OF THE FIELD EQUATIONS} The field equations for multiphase flow have been derived by many investigators, usualiy by means of applying the conservation eacurions for muss, momentum, and energy to the aynamics in some control volume. Difficulties arise in such derivations fron the manner by which cuts are made through the nidterial of one phase in order to integrate over the volutre of the other, and by the way in which area and volume integrals over a single phase are trans, formed to irtegrals arounc ur over the entire coritrol region. Ingenious techniques have been used to accomplish the averaging implied by these transformations, but controversy persists in the literature as to which procedures are corrcct. In particular. there is disagreeneni regarding the formulation of the pressure gradient ierris in the momentull equations, and controversy continues concerning the re. quirement for terms included to ensure that the fornulution is well poset.

In this section we show that an alternative type of derivation can be employed, avoiding the ambiquities that arise from control-volume techniques. We employ a biouvilie equation for the distribution of scale and velocity, and show that moments of the equation enable a relocionship to be found hetween the field properties and the detailed interactions arong phases. In addition to avoiding some of the previous derivation difficulties, the Liouville approach forms a consistent basis for the inclusion of various complicated extensions to the field equations, such as close-packed momentum transfer, the effects of a local spectrum of scales, and fluctualional pressure from the disperse phase. 
We outline a special cuse of the derivation from whicn the extended and more general furms can be construcled. The central function in the derivation is $N(r, \dot{x}, \vec{u}, t)$, which is defined in such a way that $N d r d \vec{x} d \dot{u}$ is the probable number of solid particles with size (e.g., radius) $r$ in the interval $d r$, position $\dot{x}$ within the interyal $\vec{d} \vec{x}$, and velocity $\dot{u}$ in the interual di, at time $t$. In addition, $m(r)$ is the irass of a particle with size $r\left[5.9 ., m\{r\}=4 * r^{3} r / 3\right.$, in which.: is the microscopic density of the particulate vispersed) pickse]. Fron these functions, the following manents can be formed:

$$
\begin{aligned}
& . \quad \| \text { \|ndrdu, } \\
& \cdot u_{i}=\int \| m u_{i} d r d u .
\end{aligned}
$$

and

$$
\left\|_{0}:\right\| N d r d \vec{u} .
$$

Inese functions of position and tine describe the mass of particulate slaterial per unit total voiume, . ' the mass-averaged component of velocily in direction $x_{i}, u_{i}$, and the number density of the particles. $N_{0}$. Hote that the void fraction is given $b_{j}$

$1-(, 1$,$) .$

The fundantental equation for our derivdtion expresses the conservation of the total number of particles in any volume of $r, \dot{x}, \dot{i}$ space moving with the particies. Thus we neglect fragmentation, coalescence and other processes that would alter the number of particies, the effects of which could be added as a source term to the particle-number conservation equation,

$$
\frac{d}{d t} \| \int N d r d \vec{d} d \vec{x}=0
$$

It is important to note that the total mass of particles in the arbitrary hyperspace volume is not conserved by our expression.

By means of the usual rules for jifferentiation of an integril, and the condition that the volume of integration is arbitrary. Ea. (A) can be transfurmed to the following equivalent Liouville Equation:

$$
\frac{\partial N}{i t}+\frac{\partial}{\partial x_{j}}\left(N \frac{d x_{j}}{d t}\right)+\frac{j}{\partial u_{j}}\left(N \frac{d u_{j}}{d t}\right)+\frac{j}{\partial r}\left(N \frac{d r}{d t}\right)=0
$$

in which the total time derivatives are along the dynamicaliy and kinematically allowable paths of the individual particles. Thus, for Eq. (5),

$$
\frac{d ;}{d t}=u_{j}
$$

$$
\text { iii } \frac{d u j}{d t}=F_{j},
$$

and dr/dt $i$ setermined from the rate finction for phase transition and the geometrical configuration of the particle. Assuming that the particles ore spheres, we can write

$$
\frac{d m}{d r}=a \cdot r^{2}
$$

whict: will allow the mass transfer rate to be related to the rate of change of radius. Indeed, because of the unique relationsnip in this case between $m$ and $r$, the cistribution function could as weil have been $i\{(m, \dot{x}, \dot{u}, t)$.

Enuation (5) describes the dynamics of the single-particle distribution function, and depends only on expressions for the kinematics and dynamics of a sirigle particle. Nevertheless, there are two ways by which this equation can be considered to represent the multiparticle effects of the dispersed phase. One of these is through the force function, $F_{j}$, which represents the time-varying force on a single particle, but can contain in its formulation a representation of the average effects on that particle to be expected from surrounding particles. Although this is only an approximate represertation of collective effects, which could be improved by the inclusion of integrals over a two-particle distribution function as a source to $E q$. (5), we shall see that the present version is entirely sufficient to illustrate the derivation of a correct expression for the pressure-gradient effects in the field equations for momentum. This is because the second way in which Eq. (5) represents the multiparticle 
dynamics of the dispersed phase can be demonstrated by certain selected monents of the equation.

In particular, multiply Eq. (5) by $m(r)$ and $m(r) u_{i}$ and integrate over the entire range of $r$ and $i$ values. The results are

$$
\frac{\partial e^{\prime}}{\partial t}+\frac{\partial}{3 x_{j}}\left(\cdot+u_{j}\right)+\iint m \frac{\partial}{; r}\left(N \frac{d r}{d t}\right) d r d u=0
$$

and

$$
\begin{aligned}
& \frac{\partial w^{\prime} u_{i}}{\partial t}+\frac{\partial}{\partial x_{j}} \iint m N_{i} u_{j} d r d \vec{u} \\
& +\iint m u_{i} \frac{\partial}{\partial u_{j}}\left(N F_{j} / m\right) d r d \dot{u} \\
& +\iint m u_{i} \frac{\partial}{\partial r}\left(N \frac{d r}{d t}\right) d r d \dot{u}=0 .
\end{aligned}
$$

Using Integration by parts and reducing the results." we can rearrange these mass and momentum equations to the following

$$
\frac{\partial j^{\prime}}{\partial t}+\frac{\partial}{\partial x_{j}}\left(\omega^{*} \vec{u}_{j}\right)=\int N \frac{d m}{d t} d r d \dot{u} .
$$

and

$$
\begin{aligned}
\frac{\partial \rho^{\prime} \bar{u}_{f}}{\partial t}+\frac{\partial \rho^{\prime} v_{j} u_{j}}{\partial x_{j}} & =-\frac{\partial}{\partial x_{j}} \iint N m \cdot u_{f} \cdot u_{j} d r d u \\
& +\iint N F_{f} d r d \dot{ } \\
& +\iint N u_{i} d m d r d \dot{d} .
\end{aligned}
$$

In which $5 u_{i} \equiv u_{i}-\bar{u}_{i}$ "

Equations (8) and (9) are now in the form of field equations for the dispersed phase, derived without recourse to splits or cuts of a control voi we into the domitins occupled by each phase. They neverthejess express conservation of mass and momenturb for any fixed total control velune in space that one wishes to choose. An integral of Eq. (8) over such a contral valume shows that the three terms re fer, respectively, to the rate ot change of dispersed-phase mass in the volume, the convective fiux of that mass through the cages of the volume, and the rate of mass conversion to the dispersed phase by means of phase transitions within the volunke.

We have, for example, areived at an expression for convective flux without the waplicit reuvipenkent for assuming that the volume per unie total voluike of the dispersed phase is eysul tiv lite ared per unit area open for convection. In sumilar fashion, the terms in Eq. (9) can be interpreted as the rate uf change of total dispersed-phd monenturt, the conEribution to momentum chatiqu from the mont :unvective flux, the fluctuatiunal contribution andlogous to a Reynolds stress in torlistent. flow. the effect of single-particle farces on the field mowentum. and the conversion rate to dispersed-phase momentum from phase transitions.

Eluations (y) and ( 1 : nave cons iderable patentialiry for interpretation and extensions, some of which will be reported elsewhere, for our present purpose, we limit the discus'itin to the special case in which

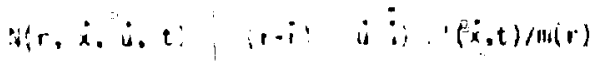

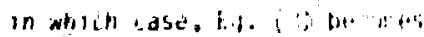

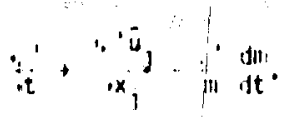

and En. (9) beconiar:

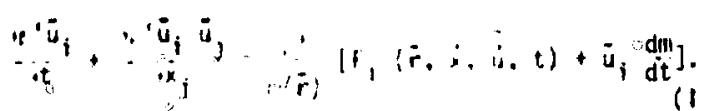

xupgose for texdmple. this!

$$
F_{i}=m g_{i}-n_{i} \mu d a
$$

in whis." $\eta_{1}$ is the wavitationet decelerdtion, $p$ is the wesisure of the curcinuous ifldse. and $n_{i}$ is an cueward unit norlid vacelor on ene particle surface at the elenent of area, da. If the low fluid velocity, $v_{i}$. diffurs froff that of the particle velority, then the pressure inteural can be expanded 
as a pawer series in $\left(v_{i}-\vec{u}_{i}\right)$, such that

$$
\begin{aligned}
F_{i} & =m g_{i}-\int n_{i} p_{0} d a+6 \pi_{\mu} \dot{r}\left(v_{i}-\vec{u}_{i}\right) \\
& +\frac{1}{2} \pi \vec{r}^{2} c_{D} i_{f}\left(v_{i}-u_{i}\right)\left(v_{i}-u_{i} i\right.
\end{aligned}
$$

In which $p_{0}$ is the pressure in the cont inuous phase In the absence of relative motion between phases.

The Stokes-drag term contains $\mu_{2}$ the fluid viscosity, and the form dragterm contains of, the fluid amity. and $C_{D}$, the drag cuefficient. Oaited are contributions such as the virtul ass teim, which dapends on the relative acceleration tetween fields. and can be systematically included in this derfvation.

If an individual particle is small compared with the scale of pressure variations in the continuous phase, then we can agroximate

$$
-\int n_{i} D_{0} d a=-\int_{3 n_{i}}^{3 p_{0}} d V=-{\stackrel{m}{3 p_{0}} .}^{3 p_{i}}
$$

Mote, however. that if the continuous phase is turbulent. then this approximation ma not be valid. In that case $\delta n_{i} p_{0}$ da mist be split into two conertbutions. one from the wean-flow pressure and the other representing the statistical effecs of the sall-scale pressure fluctuations, andloyous 20 those of Browian motion. In any case, ene transfomation in Eq. (14) is over sínale perticle. rather then set of particies in a conerol volues. and is this cructal difference iram controli. volue derlvations that allow ut to awd the dif. ficulties previously associated with chitribin the pressure-gredtent effects in the miticiose-flem. manterit rquations.

With these considarations, [n. PIII berdm

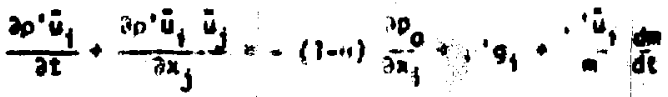

$$
\begin{aligned}
& +\frac{3 v_{1}}{2 r^{2}}\left(v_{1}-u_{1}\right)\left(\frac{3 i}{1-}\right. \\
& \left.+\frac{r_{0}}{t}\left|v_{1}-\bar{u}_{1}\right|\right)
\end{aligned}
$$

which shows the void fraction function correct? outside of the pressure gradient term.

Fo obtafn the monentum equation for the continuous phase, one writes the equation for total momentum and subtracts Eq. (15).

\section{DRIFT-FLUX APPROXJMATION}

The field equations for the fluid and particles are now written with subscripts $f$ and $p$, respective$l y$, as follows

$\frac{\partial D_{f}}{\partial t}+\frac{\partial l_{f} u_{f j}}{\partial x_{j}}=0$

$\frac{\partial x_{p}^{\prime}}{\partial t}+\frac{\partial \dot{p}^{\prime} u_{j}}{\partial x_{j}}=0$

$$
\begin{aligned}
d_{i} u_{f i} & +\frac{\partial}{\partial t}\left(\therefore f u_{f i} u_{f j}\right)=g_{i}: \dot{f} \cdot \frac{\partial p}{\partial x_{i}} \\
& +k\left(u_{p t}-u_{f i}\right)
\end{aligned}
$$

$$
\begin{aligned}
p_{i q}^{\prime} u_{p !} & +\underset{j x_{j}}{\prime} l_{p} u_{p j} u_{p j} j=g_{i} \dot{p}-(1-\cdots) \frac{j p}{j x_{i}} \\
& +k\left(u_{f j}-u_{p j}\right)
\end{aligned}
$$

in wich $k$ is a drag function. phase transitions have been neglected, and overbars plus the subscriot on $p_{0}$ have bee we now define

$$
\text { - } \cdot \dot{f} \cdot \dot{p}
$$

and

$$
+t u_{t i} \because u_{f i}+n_{p}^{\prime} u_{p i}
$$

In such a way that if fis the total mass per unit vol ume of both phases, and $u_{t i}$ is the mass average velocity so that it ${ }^{\prime} t i$ carrius the total momentum of the fluid. Then $(95$. (16) and (17) can be added to give

$\partial_{t} t_{t}+\frac{\partial f_{t} u_{t j}}{\partial x_{j}}=0$, 
and Eqs. (18) and (19) can be added to yield

$$
\frac{\partial_{i} u_{t i}}{3 t}+J_{j}\left(\partial_{f} u_{f i} u_{f j}+\partial_{p} u_{p i} u_{p j}\right)=\theta_{t} g_{i}-\frac{3 p}{3 x_{i}} .
$$

With sane manipulation, the convective flux term in parentheses can be rewritien

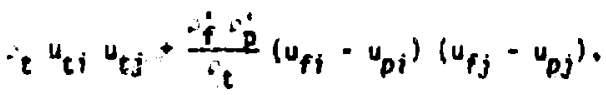

showing that the effect of relative velocity between the phases can be isolated in one term, the righthand term in Expression (22). To express this effect in a dfferent wy. the two separate momentum equations are rewriteten

$\frac{\partial u_{f i}}{\partial t}+u_{f j} \frac{3 u_{f i}}{\partial x_{j}}=g_{j}-\frac{1}{\partial f} \frac{\partial p}{3 x_{j}}+\frac{K}{n_{f}}\left(u_{p i}-u_{f i}\right)$.

and

$\frac{\partial u_{p i}}{3 t}+u_{p j} \frac{3 u_{p i}}{i x_{j}}=g_{i}-\frac{1}{{ }_{p}} \frac{ \pm p}{\partial x_{i}}+(1-.)_{i p}^{K}\left(u_{f i}-u_{p i}\right)$

In which $\therefore$ "f and if $(1 \cdots), g$. the unprimed quantities being the microscopic material densities. We subtract these equations from each other and then apply the assumption of quasi-equilibrium. occording to which the entire left side of the result is considered negligible. With some digebrdic reauction, the result is

$$
u_{p i}-u_{f i}=\frac{\cdot(1-\cdots)\left(\rho_{p}-p_{p}\right)}{K_{D_{t}}} \frac{j p}{j x_{i}}
$$

Combining Eq. (23) with Expression (22) and inserting into Eq. (21) results in the drift-flux approxi. mation for the mean momentum equation. Alterndtively. Eq. (23) car be used in Eqs. (18) and (19)

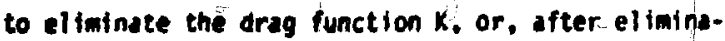
tion of the density derivatives. the results can be put in the especially simple forms

$$
\frac{\partial u_{p 1}}{\partial t}+u_{p j} \frac{\partial u_{p i}}{\partial x_{j}}=g_{i}-\frac{1}{p_{t}} \frac{\partial p}{\partial x_{j}}
$$

and

$\frac{3 u_{f j}}{\partial t}+u_{f j} \frac{i u_{f i}}{3 x_{j}}=g_{i}: \frac{1}{i x_{i}}$.

IV. STABIL:IY OF THF FQHET IONS

Consider the simplest two-phuse flow with reld-

tive velocity between the phases. namely a numogeneous configurstion in which the density of each

field is constant in space and time, and equilibrium is mintained by a balance among sriform pressure gradient, constant gravity, and a constant drag force between the materiai. As on example. such d ficu could be realized in an ided fluidized dust bed, in which the particles are suspended by tha upward motion of a fluid or gas through the field of dust. Suppuse that a one-dimensiond perturbation were introduced along the line of flow of the continuous phase. "The question arises regarding the stability of such a perturbation.

Physical redasoning suqgests that the perturits. tion amplizude would grow. Where the particles are "clumped" or closely pached together, the lluia nuse flow more rapidly through them than nesrby where the particulate density is more sparse. Bernoulli's law indicates that the pressure would be lome' in such a region of clumping. just as it is in the tliroat of d Venturi contraction. As a result. particles would accelerate towards the region of clumping and the perturbation antolitude increase. This is a longitudinal variant of the transverst: Heimhollz instadilily, which is ariven by a simildr variation in pressure along the sereamlines on either side of an irregular interface.

A detailed linear stability analysis confimis the intuitive reasoning, and shows in wore detall both the growth and propagation of such longitudinal perturbations. In the absence of viscous dissipation, the equations exhibit an instability growth rate that becomes infinite as the perturbation wave length becomes vanishincly small. As a result, the equations are classed as $1: 1$ posed. ${ }^{3}$ and therefore not applicable to the solution of initial-value problems.

In physical realfty, the ill-posed nature is precluded by dissipative processes, which are es"pecially effective in damping the highest frequency" components of any perturbation, at the same time 
naving relatively little effect on the larger scale fluctuations. Higuren illustrates such a process terough a eimparion uf instability gromeh rate as d function of perturtation wavelength for various monnituates of the viscosity coefficiert in noment um

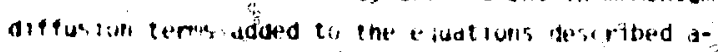
bous. dith hu dissibation, the yrowth rate is: bounded, hut at the same thine not strongl, disbilacted tron the inviscid growin cate at larger weviolengths. ine illplications of all this for the nulmerical solution of the equations is described in the next section.

\section{b. WHERICAL SOLIT:ON OF THE LOUATIO'S}

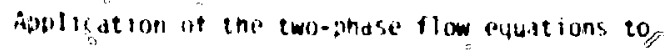
Ihe andiys is at a sper if 1 , probles' ladds fo ared?

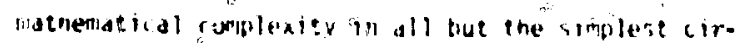
curseancer for inis reason. it has been necessar.

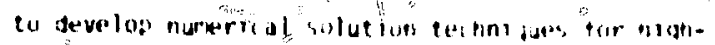

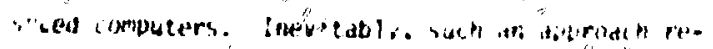

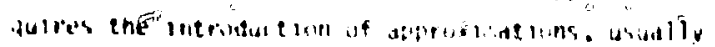

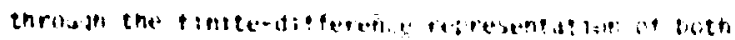

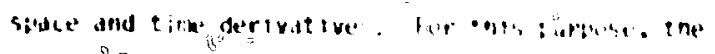

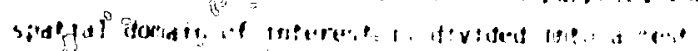

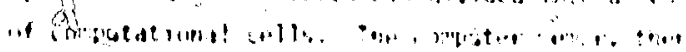

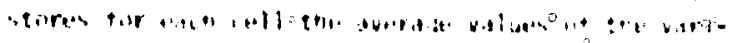

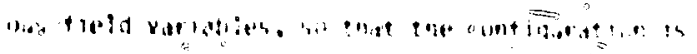

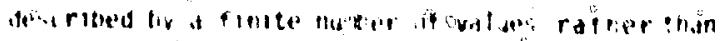

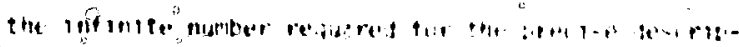

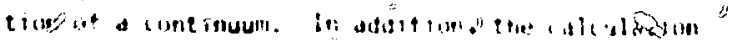

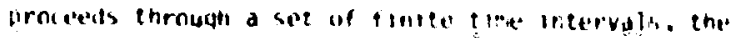
results for cidh new step being c alculated frus.

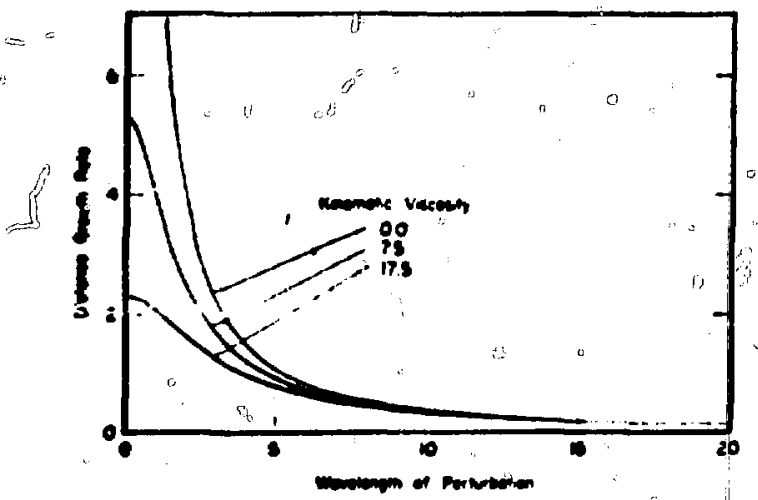

Fig. 1. "Instabllity" Grouth Rate ds" function of Perturbation Wavelength for Various kine. matic Yiscosity Coefficients. those $c \%$ the previaus, step, or from the prescribed initial conditions, by means of tou finite-differ: ence approximations to the differential equations.

Such finite-difference approach, descrived in detail elsewnere. 1.2 imediately raises wuestions concerning accuracy, for which two distinct aspects can be recognized. First there it, the question of iepresenting the true physical processtes with realistic differential equations and interaction functions for wich several coments have already been made. Second, there is the question of the errors introduced by the finite-difference approximations.

The numerical errors are of ieseral different: kinds. One arises fron coarseness of resolution, and can be mitigated by the use $0^{\circ}$ a mesh scale that cis fine compared with the sfictial scale for aporeciable changes in the fipld variables, and by time-step intervals that are sinal! compared with the ot ine scale for appreciable iocal changes. Anotner is numerical instability in whict the calculated resultes ascillate around the des red solution with increasina amplitude" or othenwise dritt away from the desired results.

Vunerical instability can sometimes be cured $n$. Hedns of an implicit formulaticn uf the finitedifference equations or by the irtroduction of a dissipative mechanism. for example. viscusity the technique we have heen using emplovs botn.

The inolicitmess is required because of our nead for flow dnalysis di very low Mach nunbers andior witn strong coupling between the fields. This crucidd feature is smphasized in the original reporis of the technique and is pot disciussed iurther in the bresent paper.

The infroduction of viscostey as a disstpdtive vrocedure mus: be accomplished with great care since numerical stability often rejulres masnitudes fur the viscosity coefficient that areatly exceed the true values for the materials. Specifically. the viscosity must not be so ared as tu Gamp excessivelly the longer wavelength perturbations described in:the previous section. Fur numarica) stability, however. the hinematic viscosity. aust usually exceed sone constant fraction of the uroduct of fluid speed $u_{0}$ and mesh.cell size. $x$, Roughly. when the time step per cyile is appropriately chosen, we may estimate for the required viscosity 


$$
=u_{0} i x .
$$

Thus, the smaller the cell size, the less viscosity is required. At the same time, fig. I shuws that the smaller the yiscosity, the smallar is the perturbation wavelength that is essentially ugaltered. Thus the two considerations are compatible. For a given physical scale to be resolved, the mesh siale, $j x$, can be reduced, at least in principle, to te extent that the colitortion is resolved and the viscosity required for numerical stability will haye $n$ igligible effect on the scale of the contortion.

In practice, this necessiry fineness of mesh siale can, indeed, be realized with present computers for many, but not all, problems of interest. The next section describes and illustrates several examples.

\section{NUMERICAL EXAYPLES}

In crder to demonstrate trat. long-wavelength: instability cun be accurately calcuiated, despite. the mitigation of very short-wavelength instability by means of numerical dissipation, we consider the configuration of a fluidized dust bed in which enysical instability is well known to occur. Figure 2 illustrates the physica! slugging flow for: such a dsst bed. The calculation commenced from slightly perturbed equilibrium and developed the oscillatory behavior in a spontaneous and nutural fashion. = Void fraction as a function of height from the floor of the bed shows a progression of propagating waves with exponentially growing amplitude (in excellent agreement with linearized stability-analys is pre-

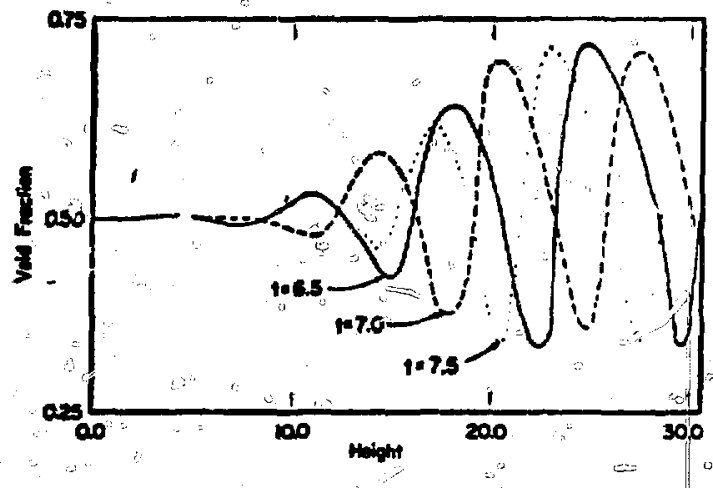

Fig. 2. Vofd Fraction vs Height from the floor of a Function of Perturbation Wavelength for Various Kiratic Viscosity Coefficients.
Gictions) in the lower rejions, and all allifilude that is bounded by nonlinear effects in thie upher regions. This example demonstrates that Ahysical instabilities can indeed be accurately calculdted with neither the disastrous planifestation, of shortwavelength instability nor excess smouthnim for the dynamics of interest.

(b) o Our second example is desiuned to examinte a possible. but nighly improbable, scenario of an axtremely simplified hypothetical liquid metal fast breeder reactor (LMFBR) disassembly accident. A iypical configuration of the core-reflector-nlenum region in cylindrical coordinates is didyratuled infiq. 3. The void contains sodium vapor while the remaining volute is filled uniformiy with granium-diunide and steel droplets in the core and steel droplets everywheré else. Due to nondissipating power incriases in the core region, high vapar pressure can develop.

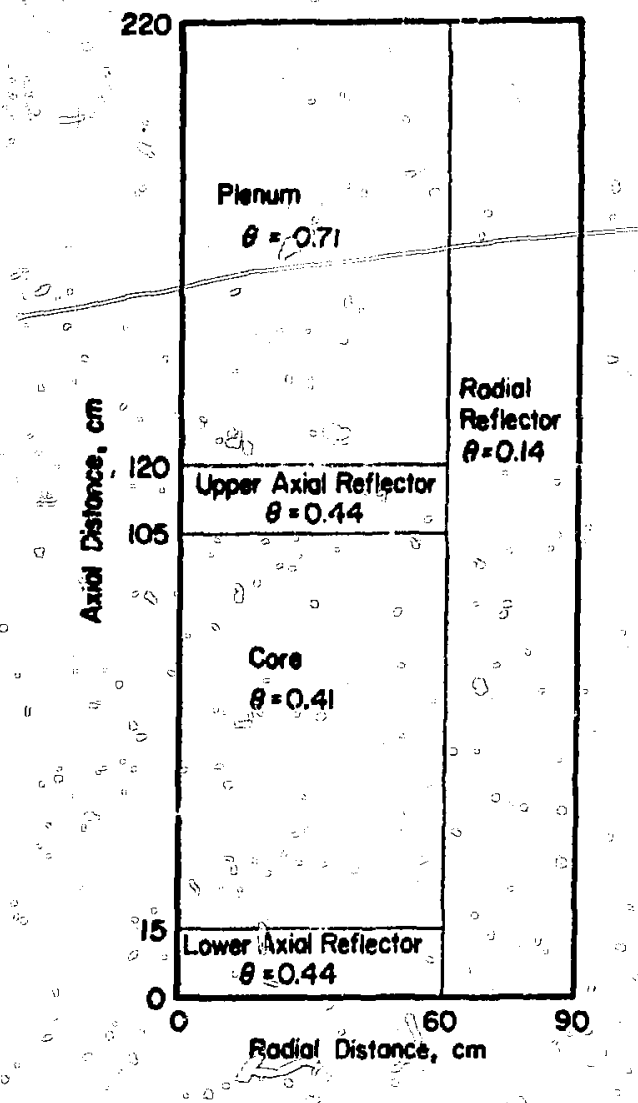

Fíg. 3. A Typtcal LMFBR Core-Reflector-Plenum Configuration. "I denotes the Vold Fraction. 
resultun in a pressure-dariven expafision. The notton dild riugt-hand boundaries are fonsidered rigid, write material is allowed to enter and exit througn the top. A compos të of the various fields and their tinke evolution is presented in Fig. 4 . The narber-particle corifiguration depicts the distribution and density of the various materidls throughout the compitational mesh. Fuel and steel droplets are represented respectively by the siuare and plus sions, while sodiuni vapor is represented ty the dots. The velocity fields a re nonnalized to the maximim velocity un the mesh for that particular field.

The calculation proqresses with an intense shock developing in front of the sodium vapor expandinin out of the pressurized ccre region. Berauce of the tight coupling setween the vapur and droplet fields. the droplet motion is il itidily almost coincident with the vapor expdnsion. The snock defortis and compresses the reflector reqions before first impacting the lower boundary and then the right-hand boundary this results in aroplets piling up against these boundaries ard developing total'y inconpressitie regions shown by the low or .. :-0.0 contour line in the void fraction field.

The shock reflects off the boundaries, and the sootium vapor sireams towards low pressure regions dragging aroplets diong. Inthe find stages of the calculation, materid is shown to be rafidly exiting the lup boundary, and the central region becomes highly voided. Al though this example represents an idealized hypothetical iMFGR disassenbly accident, it clearly demonstrates the capenility of calculdtirg interpenetrating materials in two-phase flow problems. v11. CUNCLUSIOn:s

Because most two-phase flow urotlebis are :c naturally complicated, nigुo-spete digital computer: gre being employed to solve the coubied nonlinear partial différential equations tind describe the dynamics of such a conblex physical system. Trie formulation of these equations, a" reported in the original work, is shown to be correct by a derivation fron a statistical viewpoint, which is based on the momerits of a Liouxilie equation. These cifferential field equations governing the miction of two-phase interactiogris are "well posed" as 1ilustrated by a fluidized dust bed calculation. In this a example we see that the dynamics of resolvable flow contortions can be successfully calculated without requiring excessive dissipation or smcothirig, ar.t. d the same time avaiding fire scale instabilities that otherwise would destroy the validity of the results.

\section{REFERENCES}

1. F. H. Harlow and A. A. Amsden, "Numerical Caiculation of Multiphase Fluid Flow," J. Comp.. Phys. 17. $19(1975)$.

2. A. A. imsden and F. H. Harlow, "KACHINA: "An Eulerian Computer Program for Mulifield Fluid Flows." Los Alamos Scientific Laboratary report LA- 5680 (1974).

3. Peter Lax, personal comunication. 
Is. 4. Exaple of a preseure driven diganeabily

Tine -0.0 sec

(a) Hekne-Farticle Coariguration

$$
\text { Tee }-10^{-3} \text { eec }
$$

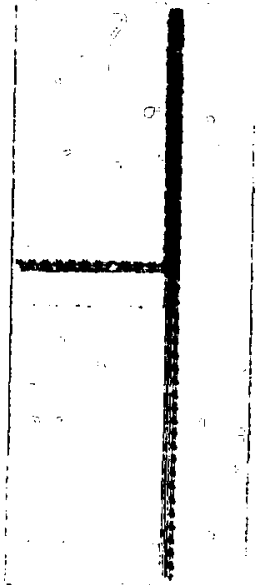

(d) Vold fraction

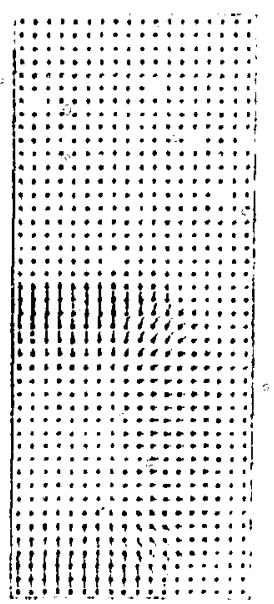

(b) Vapor velocity Field

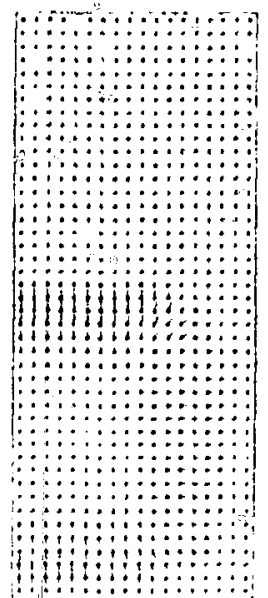

(c) Droplet velocity Field

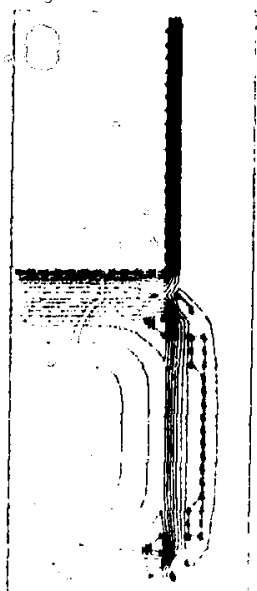

(d) Void fraction 


\section{(a) Murker-Farticle Configuration}

$$
\text { Time }-2 \times 10^{-3} \text {.ace }
$$
(b) vepor veloeity Field
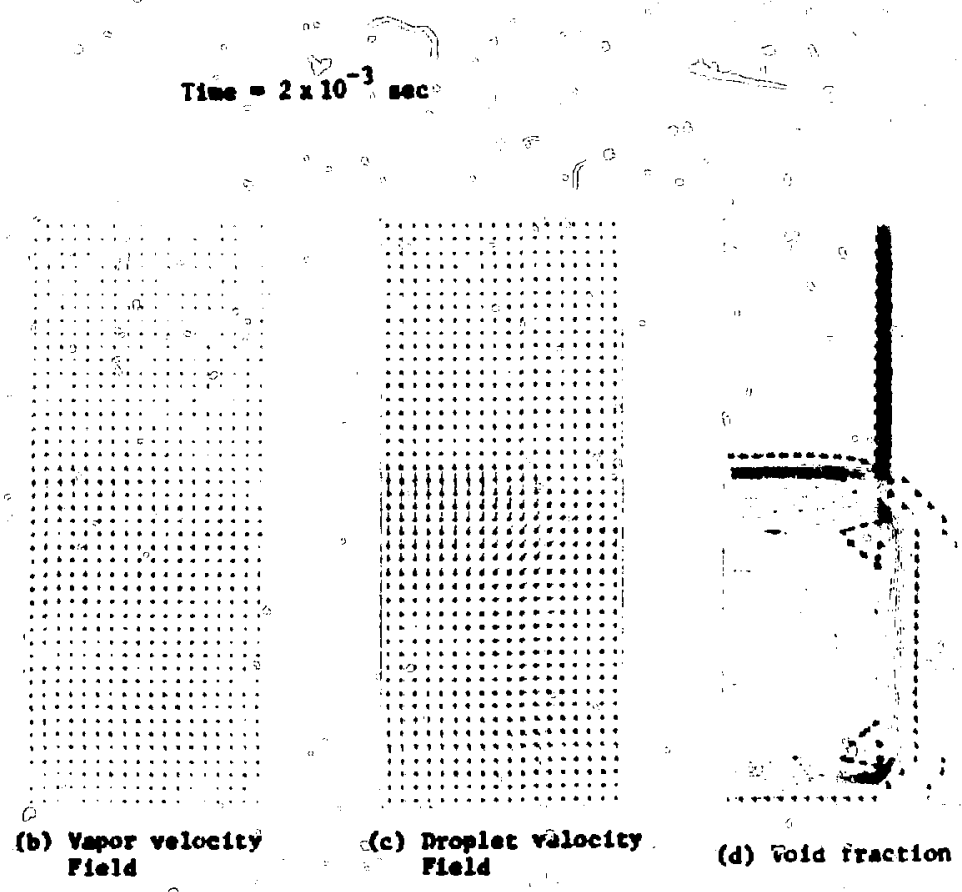

(d) Fold iraction

TIEs $-5 \times 10^{-3}$ ace

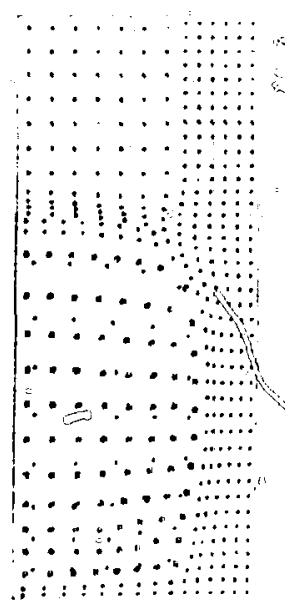

(a) Marker-Particle Conf ifuration

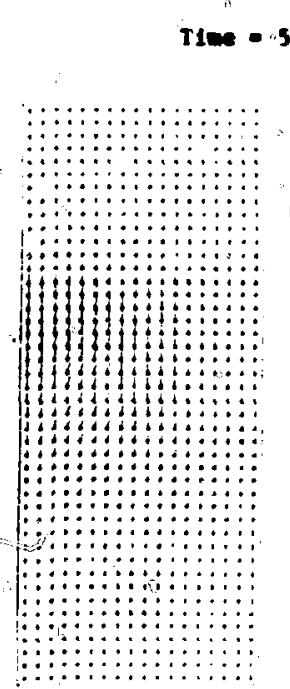

(b) Vepor velocity Field

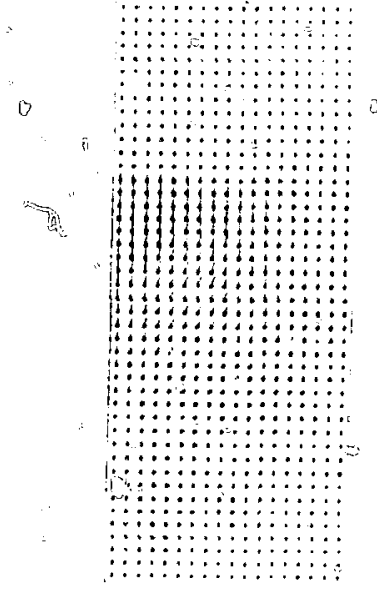

(c) Droplet velocity Field

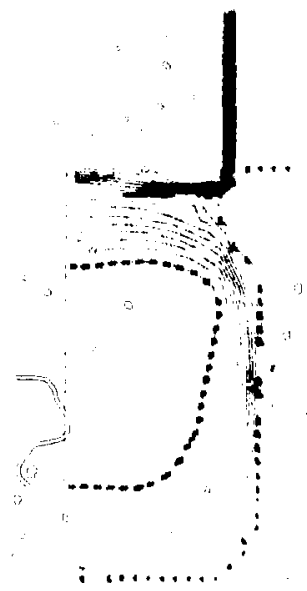

(d) Volit fuccton 
If $-15 \times 10^{-3} \sec$

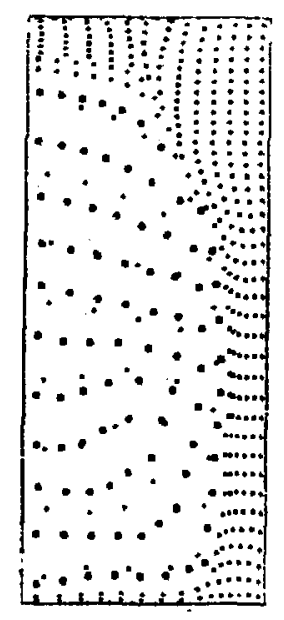

(a) Markex-Partiele Contisuration

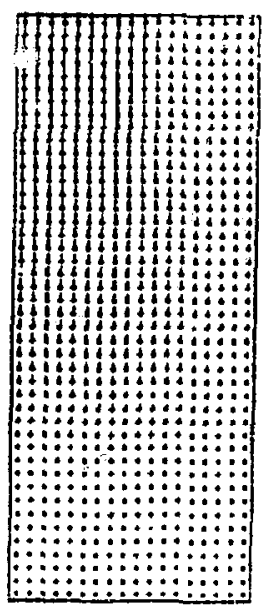

(b) Vapor velocity Feld

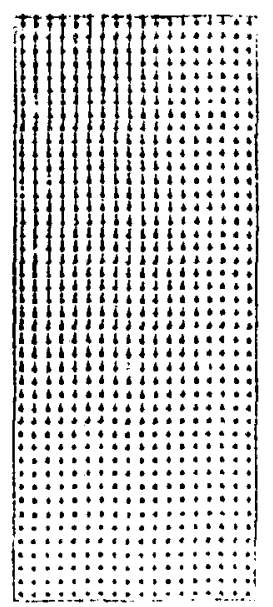

(c) Droplat veloctty

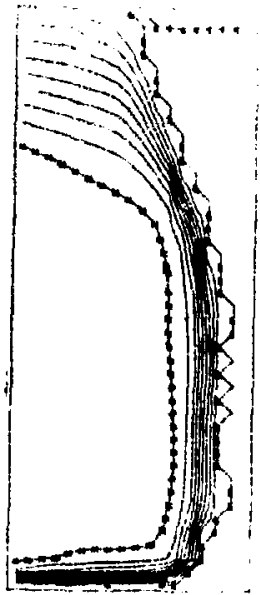

(d) Veld fraction

Tine $-30 \times 10^{-3} \sec$

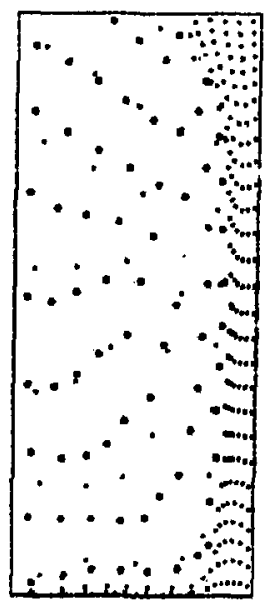

(a) Markor-Particle Conf1quration

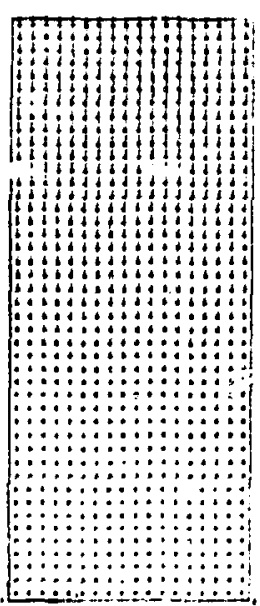

(b) Yepor veloetty Field

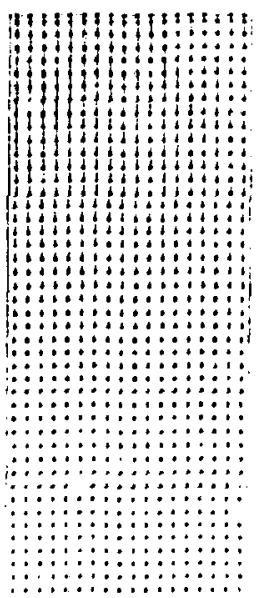

(c) Droplet veloetity Field

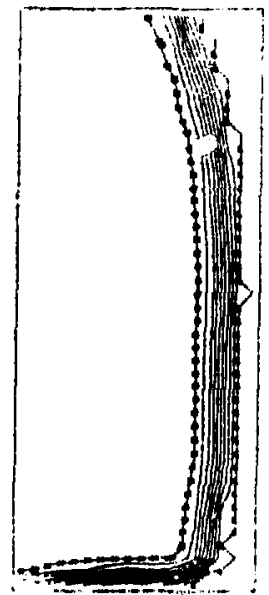

(d) Vold frwetion 